\title{
Consumo de alimentos com propriedades antioxidantes por idosos institucionalizados
}

\author{
Consumption of food with antioxidant properties institutionalized elderly
}

\author{
Mariane Rosa', Lina Cantarelli', Elisângela Colpo ${ }^{2}$ \\ ${ }^{1}$ Nutricionista pelo Centro Universitário Franciscano, Santa Maria, RS; ${ }^{2}$ Professora do Curso de Nutrição do Centro Universitário Franciscano, Mestre e Doutora em \\ Bioquímica Toxicológica pela Universidade Federal de Santa Maria (UFSM), Santa Maria, RS.
}

\section{RESUMO}

Objetivos: Analisar o consumo de micronutrientes com propriedades antioxidantes em idosos institucionalizados.

Métodos: Realizou-se um estudo transversal com residentes de uma instituição de longa permanência para idosos do sexo masculino. O consumo alimentar foi avaliado por meio de observação direta das refeições realizadas e foi calculado no software dietWin Profissional 2008 . A adequação dos macronutrientes seguiu as recomendações da Sociedade Brasileira de Alimentação e Nutrição e o consumo de micronutrientes foi analisado de acordo com a Ingestão Dietética de Referência. Os testes utilizados foram correlação de Sperman e descritiva simples. O nível de significância utilizado foi $\mathrm{p}<0,05$.

Resultados: $\mathrm{O}$ estudo incluiu 28 idosos do sexo masculino. A ingestão média de carboidratos e lipídios era adequada, porém o consumo médio de proteínas foi um pouco elevado. A ingestão média dos micronutrientes era inadequada, com exceção do consumo médio de zinco e ferro. O micronutriente com maior percentual de inadequação foi o tocoferol. Houve correlação positiva entre consumo de Tocoferol e lipídios e correlações negativas entre o consumo médio de vitamina $\mathrm{C}$ e a idade, entre o consumo médio de selênio e os níveis sanguíneos de leucócitos e entre triglicerídeos e consumo de zinco.

Conclusões: Encontrou-se inadequação no consumo da maioria dos micronutrientes com propriedades antioxidantes. É importante estabelecer estratégias de educação nutricional e adequação do cardápio dos idosos institucionalizados para corrigir essas deficiências.

DESCRITORES: INSTITUIÇÃO DE LONGA PERMANÊNCIA PARA IDOSOS; ASILO PARA IDOSOS; MICRONUTRIENTES; NUTRIÇÃO; ALIMENTAÇÃO.

\section{ABSTRACT}

Aims: To analyze the consumption of micronutrients with antioxidant properties in institutionalized elderly.

Methods: We conducted a cross-sectional study with residents of an institution for the aged male. Dietary intake was assessed by direct observation of meals and was calculated in DietWin Profisional 2008 software. Adequacy of macronutrients followed the recommendations of the Brazilian Society of Food and Nutrition and micronutrient intake was analyzed according to the Dietary Reference Intakes. The tests used were Spearman correlation and descriptive statistics. The level of significance was $\mathrm{p}<0.05$.

Results: The study included 28 elderly males. The average intake of carbohydrates and lipids was adequate but the average protein intake was slightly elevated. The average intake of micronutrients was inadequate, except for the average intake of zinc and iron. Tocopherol was the micronutrient with the highest percentage of inadequacy. There was a positive correlation between consumption of tocopherol and lipids and negative correlations between the average intake of vitamin $\mathrm{C}$ and age, between the average selenium intake and blood levels of leukocytes and between triglycerides and zinc intake.

Conclusions: The intake of most micronutrients with antioxidant properties was inadequate. It is important to establish strategies for nutrition education and adjustment of the menu of institutionalized elderly to correct these deficits.

KEY WORDS: GERIATRIC LONG-TERM CARE INSTITUTIONS; HOMES FOR THE AGED; MICRONUTRIENTS; NUTRITION; FOOD. 


\section{INTRODUÇÃO}

O envelhecimento populacional é reconhecido como fenômeno mundial que iniciou nos países desenvolvidos e estendeu-se aos menos desenvolvidos. Nos últimos anos, o Brasil apresentou um significativo aumento no número de idosos e, de acordo com a Pesquisa Nacional por Amostra de Domicílios (PNAD) realizada em 2009, o Brasil possuía, naquele ano, uma população aproximada de 21 milhões de pessoas com 60 anos ou mais de idade, o que representa $11,3 \%$ do total da população. ${ }^{1-2}$

Quando o cuidado ao idoso não pode ser realizado por seus familiares, uma alternativa de cuidado consiste em uma instituição de longa permanência para idosos (ILPI), que pode ser pública ou privada. A crescente necessidade de proporcionar abrigo aos idosos em instituições tem despertado a atenção da população de um modo geral, pois o envelhecimento e o aumento da expectativa de vida, associados à redução da capacidade física, cognitiva e mental, exigem assistência social e à saúde. ${ }^{3,4}$

No envelhecimento ocorrem mudanças fisiológicas decorrentes da passagem do tempo, as quais tornam o indivíduo mais susceptível a doenças, principalmente crônico-degenerativas, além de ocasionar o desenvolvimento de incapacidades psicomotoras. ${ }^{5}$ Dentre as teorias propostas para explicar esses processos, inclui-se a do estresse oxidativo, a qual sugere que o envelhecimento provém de danos celulares, gerados por espécies reativas ao oxigênio (EROs). ${ }^{6}$

$\mathrm{O}$ estresse oxidativo, que consiste em um desequilíbrio em prol dos compostos oxidantes e em detrimento dos agentes antioxidantes, ${ }^{7}$ contribui para o envelhecimento celular, sendo que este está relacionado aos danos provocados pela reação das EROs com DNA, RNA, proteínas e outras substâncias oxidáveis. Essa reação contribui também para a instalação de doenças, entre as quais câncer, aterosclerose e artrite reumática. ${ }^{8}$

O sistema de defesa antioxidante tem como principal função manter o processo oxidativo dentro dos limites fisiológicos e da capacidade de regulação, de modo a impedir a ampliação de danos oxidativos e a consequente formação de lesões sistêmicas irreparáveis. Dentre os importantes fatores na modulação do estresse oxidativo, está inserida a dieta, que inclui vitaminas como ácido ascórbico, retinol, tocoferol e os microelementos zinco e selênio. ${ }^{7}$

O sistema antioxidante apresenta-se incompleto sem os agentes antioxidantes provenientes da alimentação, o que ressalta a importância da ingestão diária destes componentes. O consumo de antioxidantes contribui para a melhora da saúde e da qualidade de vida. ${ }^{9}$ Desta forma, são necessários estudos sobre estes compostos, principalmente em idosos, os quais apresentam necessidades importantes de elementos antioxidantes, em decorrência do envelhecimento celular que ocorre naturalmente com o passar da idade. O objetivo deste estudo foi analisar o consumo de alimentos com propriedades antioxidantes em idosos institucionalizados.

\section{MÉTODOS}

O presente estudo corresponde a uma pesquisa de campo do tipo transversal e quantitativa. Foi realizado em uma ILPI de caráter filantrópico que recebe apenas idosos do sexo masculino, localizada na cidade de Santa Maria, Rio Grande do Sul. Pelo seu caráter caritativo, a maioria dos alimentos consumidos pelos moradores da ILPI é proveniente de doações. A pesquisa foi desenvolvida durante o período de julho a outubro de 2012.

Os critérios de inclusão consistiram em indivíduos com idade igual ou superior a 60 anos, que deambulavam e alimentavam-se por via oral. Foram excluídos idosos que apresentavam diagnóstico de insuficiência renal crônica ou outras doenças que necessitam de restrição em alguns alimentos da dieta, e aqueles que se alimentavam por meio de dietas modificadas. As informações sobre dados demográficos e alimentação foram obtidas através de anamnese, que foi respondida pelos idosos e/ou pelos funcionários e responsáveis pelo local. Além disso, foi efetuada uma consulta aos prontuários para verificar exames bioquímicos de rotina dos últimos 90 dias, incluindo hemograma, glicemia, ureia, creatinina, colesterol total e triglicerídeos.

O consumo alimentar foi avaliado por meio de observação direta por acadêmicas habilitadas do curso de Nutrição. Durante as refeições, que eram realizadas no refeitório, as acadêmicas verificaram o consumo alimentar de cada idoso. Todas as refeições foram acompanhadas, bem como lanches ou alimentos que os idosos consumiram fora das refeições oferecidas pela ILPI. A observação do consumo alimentar foi realizada duas vezes por semana, durante todas as refeições do dia, por um período de três meses, totalizando 35 dias. Esse período de observação foi determinado a fim de compreender como eram os hábitos alimentares, consumo de macro e micronutrientes com propriedades antioxidantes, bem como atividades desenvolvidas pelos idosos. As acadêmicas acompanhavam as refeições quando eram servidas pelos funcionários e o quanto eram consumidas pelos idosos, se havia sobras 
ou repetições. Além disso, os alimentos/preparações servidos pelos funcionários eram pesados, a fim de transformar a medida caseira em gramas.

O consumo alimentar dos idosos foi calculado no software dietWin Profissional 2008. A adequação dos macronutrientes foi avaliada de acordo com as recomendações da Sociedade Brasileira de Alimentação e Nutrição $(\mathrm{SBAN})^{10}$ e os micronutrientes, ácido ascórbico (vitamina $\mathrm{C}$ ), retinol (vitamina $\mathrm{A}$ ), tocoferol (vitamina E), zinco, selênio e ferro, de acordo com a Ingestão Dietética de Referência (DRI), sendo utilizada, neste estudo, a Necessidade Média Estimada (EAR). Para os micronutrientes que ultrapassaram os valores recomendados, utilizou-se como limite máximo de avaliação o Nível Tolerável de Maior Ingestão (UL). ${ }^{11}$

Após o período de observação do consumo alimentar, os idosos receberam orientações nutricionais e informações sobre a importância de uma alimentação equilibrada, da hidratação e da necessidade de exercícios físicos, o que ocorreu por meio de atividades lúdicas. Estas orientações foram elaboradas com base nos "Dez passos para uma alimentação saudável para pessoas idosas". ${ }^{12}$
O estudo foi aprovado pelo Comitê de Ética em Pesquisa do Centro Universitário Franciscano, de acordo com o número do parecer consubstanciado 52900, sendo que os participantes ou responsáveis pelos mesmos assinaram o Termo de Consentimento Livre e Esclarecido. A análise estatística dos dados foi realizada através do programa Statistica 7.0 e os testes utilizados foram correlação de Sperman e descritiva simples. Os dados foram considerados estatisticamente significativos quando $\mathrm{p}<0,05$.

\section{RESULTADOS}

A amostra da pesquisa foi constituída por 28 idosos com idade entre 61 e 99 anos, sendo a média de idade de 73,8 89,4 anos. A média do valor calórico total diário dos idosos foi de $1837,5 \pm 562,8 \mathrm{kcal}$, sendo o percentual de consumo médio de carboidra tos $62,15 \pm 10,3 \%(1137,26 \pm 383,4 \mathrm{kcal})$, de proteínas $15,46 \pm 4,4 \%(283,6 \pm 120,7 \mathrm{kcal})$ e de lipídios $22,4 \pm 8,3 \%(416,3 \pm 207,7 \mathrm{kcal})$.

Ao analisar o consumo de vitaminas, a ingestão média diária de ácido ascórbico foi $65,9 \pm 41,7 \mathrm{mg}$, de retinol foi $3633,8 \pm 8141,9 \mu \mathrm{g}$ e de tocoferol $5,9 \pm 7,8 \mathrm{mg}$.

Tabela 1. Adequação do consumo de micronutrientes em idosos do gênero masculino, com idade entre 61 e 99 anos, residentes em uma instituição de longa permanência de caráter filantrópico. Santa Maria, RS, 2012

\begin{tabular}{|c|c|c|c|}
\hline Micronutriente & Consumo médio diário \pm desvio padrão & \% Adequação (EAR) & Valores de Referência (EAR)** \\
\hline Vitamina C & $65,9 \pm 41,7 \mathrm{mg}$ & 87,87 & $75 \mathrm{mg}$ \\
\hline Vitamina $\mathrm{E}$ & $5,9 \pm 7,8 \mathrm{mg}$ & 49,17 & $12 \mathrm{mg}$ \\
\hline Vitamina A & $3633,8 \pm 8141,9 \mu \mathrm{g}$ & $121,13^{*}$ & $3000 \mu g^{*}$ \\
\hline Zinco & $10,3 \pm 3,5 \mathrm{mg}$ & 109,57 & $9,4 \mathrm{mg}$ \\
\hline Selênio & $40,3 \pm 14,6 \mu \mathrm{g}$ & 89,56 & $45 \mu \mathrm{g}$ \\
\hline Ferro & $10,6 \pm 3,9 \mathrm{mg}$ & 176,67 & $6 \mathrm{mg}$ \\
\hline
\end{tabular}

* Tolerable Upper Intake Level (UL).

** Fonte: Dietary Reference Intakes (DRI'S). ${ }^{11}$

Tabela 2. Parâmetros bioquímicos sanguíneos em idosos do gênero masculino, com idade entre 61 e 99 anos, residentes em uma instituição de longa permanência de caráter filantrópico. Santa Maria, RS, 2012

\begin{tabular}{|lcc|}
\hline Parâmetros Bioquímicos & $\begin{array}{c}\text { Média } \begin{array}{c}\text { desvio padrão } \\
(\mathbf{n = 2 8})\end{array} \\
\text { Leucócitos }\left(\mathrm{mm}^{3}\right)\end{array}$ & $\begin{array}{c}\text { Valores de } \\
\text { Referência* }\end{array}$ \\
\hline Hematócrito $(\%)$ & $8044,2 \pm 2683,2$ & $4000-11000$ \\
\hline Hemoglobina $(\mathrm{g} / \mathrm{dL})$ & $43,5 \pm 3,5$ & $40-54$ \\
\hline Glicemia de jejum (mg/dL) & $13,8 \pm 0,9$ & $13,5-18$ \\
\hline Ureia $(\mathrm{mg} / \mathrm{dL})$ & $87,5 \pm 28,8$ & $60-99$ \\
\hline Creatinina $(\mathrm{mg} / \mathrm{dL})$ & $38,7 \pm 17,5$ & $10-40$ \\
\hline Colesterol Total $(\mathrm{mg} / \mathrm{dl})$ & $1,09 \pm 0,3$ & $0,7-1,6$ \\
\hline Triglicerídeos $(\mathrm{mg} / \mathrm{dl})$ & $200 \pm 40,7$ & $<200$ \\
\hline
\end{tabular}

* Fonte: Calixto-Lima; Reis. ${ }^{13}$
Em relação à ingestão de microelementos, o consumo médio diário de zinco foi $10,3 \pm 3,5 \mathrm{mg}$, de selênio foi $40,3 \pm 14,6 \mu \mathrm{g}$ e do mineral ferro $10,6 \pm 3,9 \mathrm{mg}$. Os valores estimados de consumo de vitaminas e micronutrientes, assim como os valores considerados adequados estão descritos na Tabela 1 .

O consumo diário de ácido ascórbico apresentou $12,13 \%$ de inadequação em relação aos valores preconizados pela EAR, o tocoferol apresentou 50,83\% de inadequação e o selênio $10,44 \%$ de inadequação. O consumo médio diário de zinco, de ferro e de retinol ficou acima da recomendação de EAR, conforme a Tabela 1.

Os parâmetros bioquímicos sanguíneos estão descritos na Tabela 2. Observa-se que todos os parâmetros 
analisados estão dentro dos valores de normalidade, com exceção dos valores séricos de ureia, hemoglobina e colesterol total, que estão próximos do limite de normalidade.

Observou-se uma correlação negativa significativa entre a idade dos idosos e o consumo de ácido ascórbico $(\mathrm{r}=-0,39 ; \mathrm{p}=0,0387)$, conforme exposto na Figura 1. Verificou-se correlação positiva entre consumo de retinol e de lipídios $(\mathrm{r}=0,37 ; \mathrm{p}=0,05)$ e correlação negativa significativa entre consumo de retinol e de carboidratos $(\mathrm{r}=-0,46 ; \mathrm{p}=0,01)$. Houve correlação positiva significativa entre consumo de lipídios e

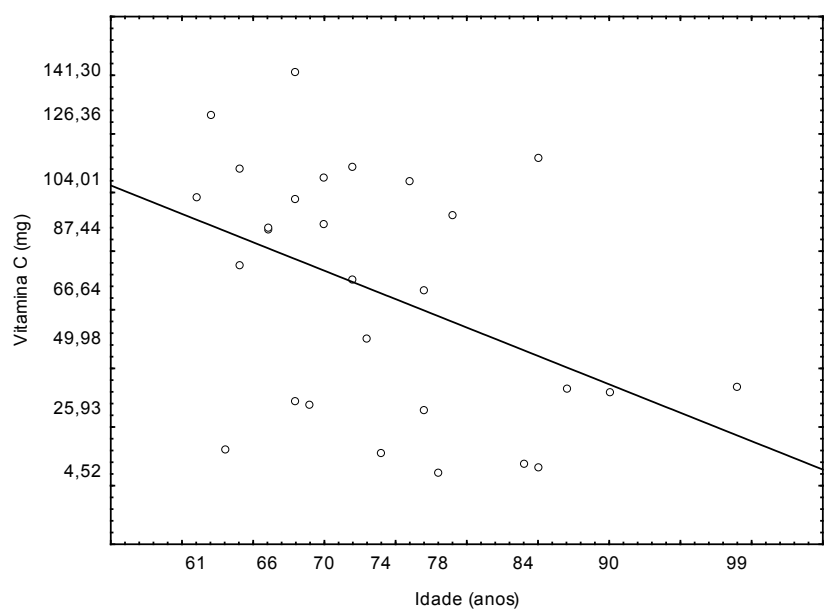

Figura 1. Correlação entre a média do consumo de vitamina $\mathrm{C}$ e a idade em idosos de uma instituição de longa permanência de caráter filantrópico. $p<0,05$. Correlação de Sperman.

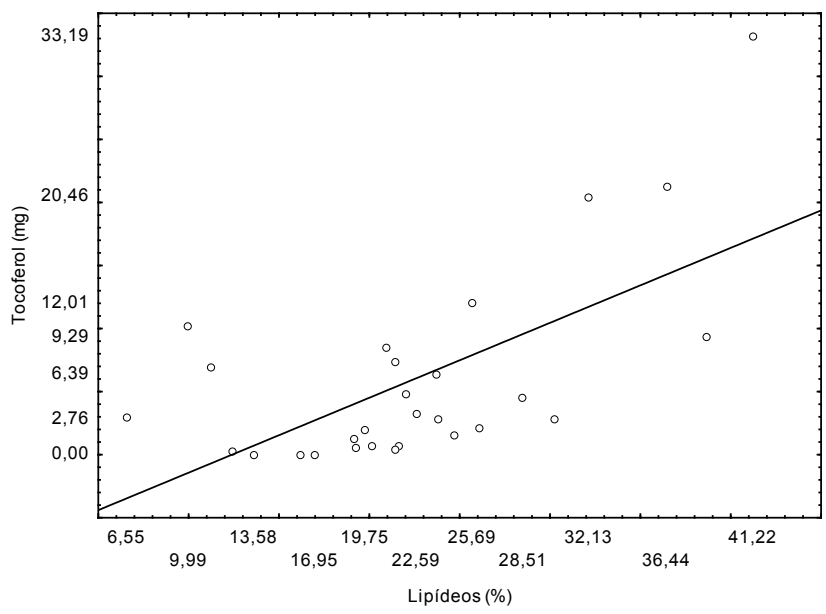

Figura 2. Correlação entre as médias do consumo de Tocoferol e lipídios em idosos de uma instituição de longa permanência de caráter filantrópico. $p<0,05$. Correlação de Sperman. consumo de tocoferol $(\mathrm{r}=0,52 ; \mathrm{p}=0,004)$, conforme a Figura 2.

Observou-se, ainda, correlação positiva significativa entre consumo de tocoferol e da vitamina colecalciferol ( $r=0,68 ; p=0,000076)$. Houve correlação negativa significativa entre o consumo de selênio e níveis sanguíneos de leucócitos $(\mathrm{r}=-0,45 ; \mathrm{p}=0,027)$, conforme a Figura 3. Observou-se correlação positiva significativa entre consumo de zinco e de ferro $(r=0,53$; $\mathrm{p}=0,003$ ), bem como correlação negativa significativa entre consumo de zinco e valores de triglicerídeos $(\mathrm{r}=-0,58 ; \mathrm{p}=0,01)$, conforme demonstra a Figura 4 .

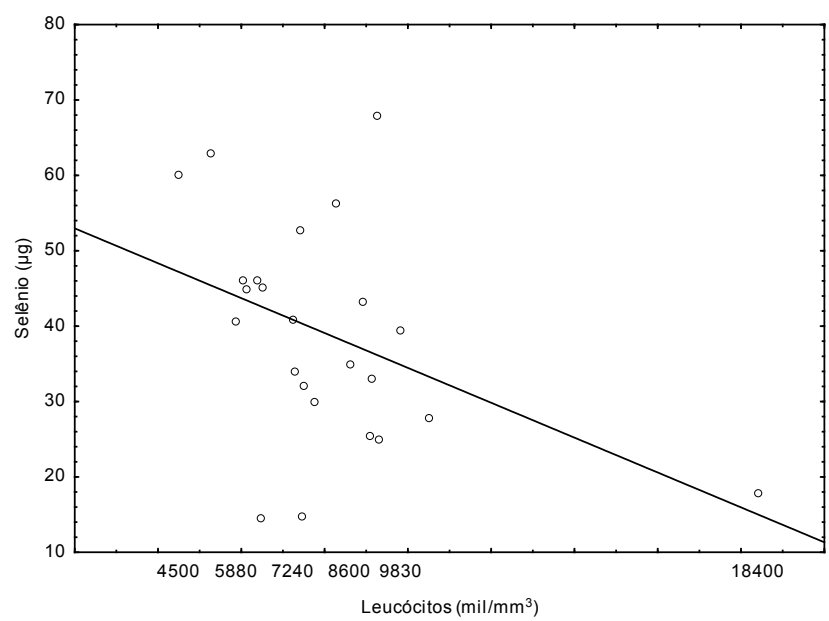

Figura 3. Correlação entre as médias do consumo de selênio e níveis sanguíneos de leucócitos em idosos de uma instituição de longa permanência de caráter filantrópico. $p<0,05$. Correlação de Sperman.

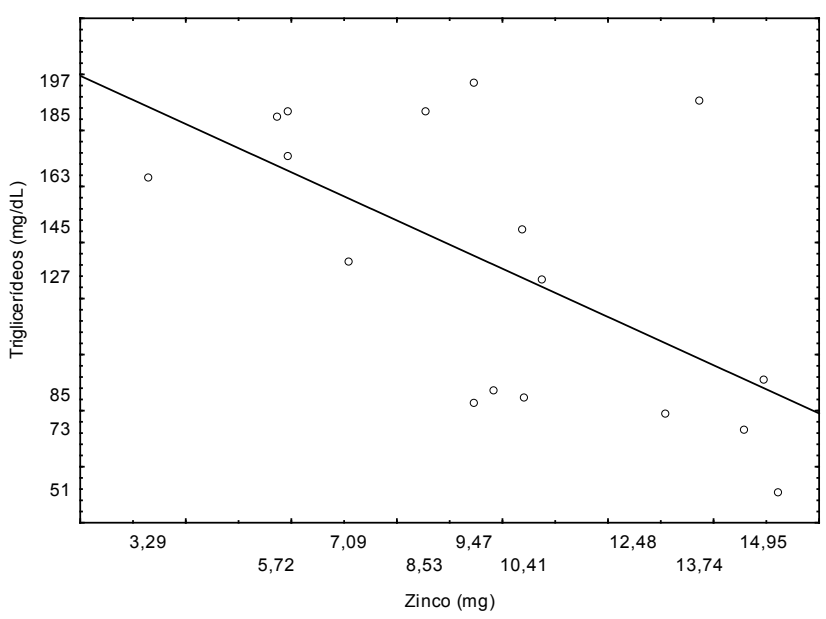

Figura 4. Correlação entre triglicerídeos e a média do consumo de zinco por idosos de uma instituição de longa permanência de caráter filantrópico. $p<0,05$. Correlação de Sperman. 


\section{DISCUSSÃO}

Em relação aos macronutrientes, o consumo médio de carboidratos e lipídios pelos idosos avaliados estava adequado, enquanto o de proteínas foi um pouco elevado, em comparação aos valores propostos pela SBAN. Esta preconiza, para idosos, 60 a $70 \%$ de carboidratos, 10 a $12 \%$ de proteínas e 20 a $25 \%$ de lipídios. ${ }^{10}$

Uma pesquisa realizada por Pont em 2009, com 33 idosos de ambos os sexos que participavam do "Programa de Atendimento Multidisciplinar à Saúde do Idoso" de Criciúma, Santa Catarina, verificou que os valores encontrados para macronutrientes apresentaram-se adequados, resultado semelhante ao presente estudo, exceto em relação à adequação de proteína. Nessa mesma pesquisa, o autor refere que o consumo adequado de macronutrientes proporciona benefícios à saúde dos idosos, como a utilização de carboidratos como fonte de energia, a síntese proteica, bem como o fornecimento de ácidos graxos essenciais e vitaminas lipossolúveis, funções de suma importância à vida humana. ${ }^{14}$

Observou-se que a média do consumo de micronutrientes está inadequada, com exceção da ingestão de zinco e de ferro que pode ser considerada adequada. $\mathrm{O}$ alto valor de retinol estimado na dieta pode ser explicado pela presença de bife de fígado no cardápio, principal fonte dessa vitamina. No entanto, o bife de fígado é servido com uma frequência média de uma a duas vezes ao mês. Outras fontes de vitamina A, como ovos, moranga e batata-doce, são encontradas com frequência na alimentação dos idosos, contudo esses alimentos, quando consumidos, não chegam a ultrapassar as necessidades diárias.

Corroborando com a estimativa de adequação no consumo médio de ferro, os valores de hematócrito e hemoglobina dos idosos indicavam ausência de anemia. Este foi um resultado satisfatório em relação à ILPI pesquisada, visto que vários estudos apontam a inadequação na ingestão desse mineral por idosos. ${ }^{15}$ Em concordância com o estudo realizado por Menezes et al., ${ }^{15}$ as principais fontes de ferro na alimentação dos idosos estudados foram o feijão e a carne bovina.

Observou-se ainda que, quanto maior o consumo de alimentos ricos em vitamina $\mathrm{A}$, maior era o consumo de lipídios e menor o consumo de carboidratos da dieta. Acredita-se que isso possa ser explicado pela predominância da ingestão de fontes concomitantes de retinol e lipídios, em detrimento das fontes de carboidratos. Os alimentos consumidos pelos idosos com maior quantidade de retinol e que também possuem lipídios são vísceras, especialmente fígado de animais, além do leite e seus derivados e da gema de ovos. ${ }^{16}$

A vitamina A é um importante composto antioxidante, estando relacionada à redução do estresse oxidativo e de doenças crônicas degenerativas. ${ }^{17}$ Além disso, possui funções importantes no crescimento e na diferenciação celular, e tem apresentado ação preventiva no desenvolvimento de alguns tumores, como de bexiga, mama, estômago e pele, em estudos desenvolvidos com animais. Pesquisas demonstram que o consumo regular de alimentos ricos em vitaminas A e C pode reduzir a incidência de câncer retal e de cólon. ${ }^{7}$ $\mathrm{O} \beta$-caroteno se destaca por sua ação antioxidante e é o mais importante precursor de vitamina $\mathrm{A}$, sendo que está amplamente distribuído nos alimentos. ${ }^{18}$

Demonstra-se na literatura que o consumo de vitamina $\mathrm{C}$ diminui com o decorrer da idade. Um estudo realizado com 112 idosos institucionalizados no município de João Pessoa, Paraíba, observou que 43 idosos com idade entre 60 e 93 anos apresentaram hipovitaminose $\mathrm{C}$, sendo que 26 estavam com deficiência moderada e 17 com deficiência grave. Por meio da análise do consumo alimentar, o mesmo estudo verificou uma baixa ingestão de vitamina $\mathrm{C}$, o que era previsto devido ao baixo consumo de frutas e hortaliças. ${ }^{19}$ Embora níveis sanguíneos de vitamina $\mathrm{C}$ não tenham sido quantificados no presente estudo, o resultado da análise do consumo alimentar de vitamina $C$ vai ao encontro do estudo citado.

O envelhecimento está relacionado ao dano oxidativo nas células e, com isso, faz-se necessária a presença de compostos antioxidantes. A vitamina $\mathrm{C}$ é um micronutriente essencial aos seres humanos, pois atua como agente antioxidante, neutralizador de EROs e nutriente para as células, protegendo-as de danos gerados por oxidantes. A mesma ação é realizada pelo $\alpha$-tocoferol e pelo $\beta$-caroteno. ${ }^{20}$

Verificou-se que, quanto maior o consumo de vitamina E, maior a ingestão de lipídios e de colecalciferol. Isso também pode ter relação com as fontes alimentares, visto que o tocoferol é encontrado em alimentos ricos em lipídios, como óleos vegetais, leite, gema de ovo e fígado, e o colecalciferol em gema de ovo, fígado, peixes gordurosos, leite, manteiga e margarina enriquecidos..$^{21-22}$ Dentre estes, os peixes são os únicos que não eram consumidos com frequência na ILPI estudada, devido ao seu custo maior e por não ser um alimento comumente doado.

O consumo de lipídios não ultrapassou as recomendações, entretanto o consumo de vitamina E está insuficiente, sendo que esse micronutriente apresentou 
o maior percentual de inadequação. De acordo com Batista et al., ${ }^{21}$ os alimentos com alto teor de lipídios, como o leite e a margarina, ou aqueles ingeridos com outros alimentos ricos em lipídios, poderiam ser fortificados com vitamina $\mathrm{E}$, visto que são capazes de proporcionar maior absorção desse nutriente. Além disso, sua utilização como antioxidante na indústria alimentícia demonstra ser uma alternativa eficaz para aumentar a ingestão diária desse micronutriente. A ação antioxidante da vitamina E está relacionada à inibição da lipoperoxidação das membranas celulares, o que impede a deterioração de ácidos graxos importantes ao organismo. Além dos benefícios proporcionados à saúde humana, a capacidade antioxidante da vitamina E minimiza a formação de EROs quando adicionada aos alimentos, o que melhora a qualidade nutricional dos mesmos. ${ }^{21}$

Um dado interessante obtido neste estudo consiste na relação negativa entre selênio e valores de leucócitos. O selênio é um micronutriente que possui importante atividade antioxidante por meio da sua associação com a enzima glutationa peroxidase. ${ }^{23}$ Esse mineral contribui para um bom funcionamento do sistema imunológico e melhora tanto a imunidade inata quanto adaptativa, sendo que os leucócitos fazem parte desse sistema e apresentam número aumentado em processos infecciosos..$^{24}$ No entanto, é importante ressaltar que a média de leucócitos sanguíneos dos idosos estava dentro dos padrões de normalidade.

A relação entre o consumo de zinco e níveis séricos de triglicerídeos pode estar associada ao fato de que, uma alimentação rica em alimentos refinados, importante fator de elevação dos triglicerídeos, está sujeita a deficiência de zinco, visto que, dentre as principais fontes desse mineral, incluem-se carnes bovinas, aves, peixes, leite, queijos e cereais de grãos integrais. ${ }^{22}$ Também se observou que, quanto maior o consumo de zinco, maior era o consumo de ferro, o que se deve, provavelmente, às principais fontes desses minerais se equivalerem, pois são de origem animal. Este resultado está em acordo com o consumo elevado de proteínas conforme a SBAN, o que também é corroborado pelos níveis sanguíneos de colesterol total, ureia e creatinina, próximos ao limite de normalidade. Deste modo, sugere-se que havia um consumo aumentado de proteína de origem animal pelos idosos.

Estudos indicam que o zinco, quando em concentrações adequadas, reduz a susceptibilidade do idoso a infecções, sendo um elemento importante na eficácia do sistema imune, na atividade antioxidante e na diferenciação celular durante o processo de envelhecimento. ${ }^{25} \mathrm{O}$ ferro possui grande importância no organismo e suas maiores fontes são provenientes da dieta. Esse mineral participa da síntese da hemoglobina, pigmento presente nas hemácias que permite o transporte de oxigênio e da mioglobina constituinte dos músculos. ${ }^{26}$ Além disso, é fundamental para a síntese de DNA e para o metabolismo energético. ${ }^{27}$

A insuficiência alimentar da maior parte dos micronutrientes, como a encontrada neste estudo, indica a necessidade de implementar estratégias de educação nutricional que informem sobre os benefícios desses nutrientes, além de promover adequações no cardápio que contemplem todas as necessidades nutricionais, medida importante para a saúde e melhora da qualidade de vida dos idosos. Salienta-se a importância de cada ILPI contar com a presença de um nutricionista capaz de estabelecer alternativas para o adequado cuidado nutricional dos idosos institucionalizados.

\section{REFERÊNCIAS}

1. Mendes MRSSB, Gusmão JL, Faro ACM, Leite RCBO. A situação social do idoso no Brasil: uma breve consideração. Acta Paulista Enferm. 2005;18(4):422-6.

2. Instituto Brasileiro de Geografia e Estatística (IBGE). Síntese de Indicadores Sociais: Uma Análise das Condições de Vida da População Brasileira. [Internet]. Rio de Janeiro: Ministério do Planejamento, Orçamento e Gestão; Instituto Brasileiro de Geografia e Estatística; Diretoria de Pesquisas; Coordenação de População e Indicadores Sociais; 2010. [Cited 2012 Oct 16]. Available from: http://www.ibge. gov.br/home/estatistica/populacao/condicaodevida/indicadoresminimos/sinteseindicsociais2010/SIS 2010.pdf

3. Camarano AA, Kanso S. As instituições de longa permanência para idosos no Brasil. Rev Bras Est Popul. 2010;27(1):233-35.

4. Perlini NMOG, Leite MT, Furini AC. Em busca de uma instituição para a pessoa idosa morar: motivos apontados por familiares. Rev Esc Enferm USP. 2007;41(2):229-36.

5. Gil L, Siems W, Mazurek B, Gross J, Schroeder P, Voss P, Grune T. Age-associated analysis of oxidative stress parameters in human plasma and erythrocytes. Free Radical Res. 2006;40(5):495-505.

6. Cancela DMG. O processo de envelhecimento. [Internet]. Portugal; 2007 [cited 2008 Nov 27]. Available from: http://www.psicologia. pt/artigos/textos/TL0097.pdf

7. Barbosa KBF, Costa NMB, Alfenas RCG, De Paula SO, Minim VPR, Bressan J. Estresse oxidativo: conceito, implicações e fatores modulatórios. Rev Nutr. 2010;23(4):629-43. 
8. Melo EA, Maciel MIS, Lima VLAG, Leal FLL, Caetano ACS, Nascimento RJ. Capacidade antioxidante de hortaliças usualmente consumidas. Ciênc Tecnol Aliment. 2006;26(3):639-44.

9. Ratnam DV, Ankola DD, Bhardwaj V, Sahana DK, Kumar MN. Role of antioxidants in prophylaxis and therapy: A pharmaceutical perspective. J Control Release. 2006;113(3):189-207.

10. 10. Sociedade Brasileira de Alimentação e Nutrição (SBAN). Recomendações nutricionais aplicadas à população brasileira. São Paulo: SBAN; 1990.

11. Institute of Medicine. Dietary Reference Intakes (DRI'S): Tables and Application. [Internet]. National Academy of Sciences; 2001. [Cited 2012 Nov 27]. Available from: http:/www.iom.edu/Activities/Nutrition/SummaryDRIs/DRI-Tables.aspx

12. Brasil. Ministério da Saúde. Secretaria de Atenção à Saúde. Departamento de Atenção Básica. Alimentação saudável para a pessoa idosa: Um manual para profissionais de saúde. [Internet]. Brasília: 2009 (Série A. Normas e Manuais Técnicos). [Cited 2012 Oct 30]. Available from: http://bvsms.saude.gov.br/bvs/publicacoes/alimentacao_saudavel_idosa_profissionais_saude.pdf

13. Calixto-Lima L, Reis NT. Interpretação de exames laboratoriais aplicados à Nutrição Clínica. Rio de Janeiro: Rubio; 2012.

14. Pont JMD. Programa de atendimento multidisciplinar a saúde do idoso: avaliação do estado nutricional e do consumo alimentar. [projeto de pesquisa]. Criciúma: Universidade do Extremo Sul Catarinense; 2009

15. Menezes TN, Marucci MFN, Holanda IMM. Ingestão de cálcio e ferro alimentar por idosos residentes em instituições geriátricas de Fortaleza, CE. Rev Saúde Com. 2005;1(2):100-9

16. Almeida ER, Carvalho AT, Nilson EAF, Coutinho JG, Ubarana JA. Avaliação participativa do Programa Nacional de Suplementação de Vitamina A em um município da região Nordeste do Brasil. Cad. Saúde Pública. 2010;26(5):949-60.

17. Gomes MM, Saunders C, Accioly E. Papel da vitamina A na prevenção do estresse oxidativo em recém-nascidos. Rev Bras Saúde Mater Infant. 2005;5(3):275-82.

18. Bianchi MLP, Antunes LMG. Radicais livres e os principais antioxidantes da dieta. Rev Nutr. 1999;12(2):123-30.

19. Aranha FQ, Moura LSA, Simões MOS, Barros ZF, Quirino IVL, Metri JC, Barros JC. Normalização dos níveis séricos de ácido ascórbico por suplementação com suco de acerola (Malpighia glabra L.) ou farmacológica em idosos institucionalizados. Rev Nutr. 2004;17(3): 309-17.

20. Padh H. Vitamin C: newer insights into its biochemical functions. Nutr Rev. 1991;49(3):65-70.

21. Batista ES, Costa AGV; Pinheiro-Sant'ana HM. Adição da vitamina E aos alimentos: implicações para os alimentos e para a saúde humana. Rev Nutr. 2007;20(5):525-35

22. Bueno AL, Czepielewski MA. Micronutrientes envolvidos no crescimento. Rev HCPA. 2007;27(3):47-56.

23. Ferreira KS, Gomes JC, Bellato CR, Jordão CP. Concentrações de selênio em alimentos consumidos no Brasil. Rev Panam Salud Públ. 2002;11(3):172-7.

24. Wang C, Wang H, Luo J, Hu Y, Wei L, Duan M, He H. Selenium deficiency impairs host innate immune response and induces susceptibility to Listeria monocytogenes infection. BMC Immunol. 2009;10(55):1-12.

25. Mocchegiani E, Giacconi R, Cipriano C, Costarelli L, Muti E, Tesei S, Giuli C, Papa R, Marcellini F, Mariani E, Rink L, Herbein G, Varin A, Fulop T, Monti D, Jaite J, Dedoussis G, Gonos ES, Trougakos IP, Malavolta M. Zinc, metallothioneins, and longevity-effect of zinc supplementation: zincage study. Ann N Y Acad Sci. 2007;1119:129-46.

26. Szarfarc SC, Brunken GS. Ferro: metabolismo, excesso e toxicidade e recomendações. Sociedade Brasileira de Alimentação e Nutrição. Cad Nutr. 1999;18:23-34.

27. Grotto HZW. Metabolismo do ferro: uma revisão sobre os principais mecanismos envolvidos em sua homeostase. Rev Bras Hematol Hemoter. 2008;30(5):390-7. 